\title{
INSUFICIÊNCIA DO CONCEITO MINERALISTA PARA EXPRESSAR A FERTILIDADE DO SOLO PERCEBIDA PELAS PLANTAS CULTIVADAS NO SISTEMA PLANTIO DIRETO ${ }^{(1)}$
}

\author{
Margarete Nicolodi ${ }^{(2)}$, Clesio Gianello( ${ }^{(3)}$, Ibanor Anghinoni ${ }^{(3)}$, \\ Jacques Marré ${ }^{(3)} \&$ João Mielniczuk ${ }^{(3)}$
}

\begin{abstract}
RESUMO
Fertilidade do solo é a capacidade deste de fornecer nutrientes, em quantidade e proporção adequadas para o desenvolvimento e produtividade das plantas, na ausência de elementos tóxicos. Este conceito, oriundo da teoria mineralista, é amplamente utilizado no mundo. Com a mudança do sistema de cultivo do solo do convencional (SC) para o plantio direto (SPD), é freqüente a obtenção de alta produtividade das culturas no SPD com valores de indicadores da fertilidade considerados inadequados para o crescimento e desenvolvimento das plantas no SC. É possível que a avaliação tradicional e, conseqüentemente, esse conceito não sejam adequados e, ou, suficientes, para expressar a fertilidade percebida pelas plantas em solos cultivados há longo tempo no SPD. Neste trabalho, objetivou-se avaliar a capacidade do conceito mineralista, e de sua avaliação, em expressar a fertilidade percebida por plantas de milho (Zea mays) em solo cultivado por longo período de tempo no SPD com diferentes rotações de culturas. As avaliações, por indicadores da fertilidade do solo e por rendimento de grãos, foram feitas na safra de verão 2005/2006, em dois experimentos irrigados, conduzidos há mais de 20 anos em Eldorado do Sul, RS, com diferentes históricos de cultivo. Os resultados evidenciaram que nem sempre os indicadores químicos da fertilidade do solo detectam a mudança na capacidade produtiva do solo promovida pelos diferentes sistemas de cultivo e rotações de culturas. Esses indicadores tiveram, em geral, baixo grau de associação com o rendimento das plantas. A avaliação e, conseqüentemente, o conceito mineralista da fertilidade, utilizado para definir a capacidade de um solo produzir abundantemente, foram insuficientes para expressar a fertilidade percebida pela cultura de milho em solos cultivados há longo tempo no SPD.
\end{abstract}

Termos de indexação: indicadores químicos, rendimento de grãos, tempo de SPD.

\footnotetext{
(1) Parte da Tese de Doutorado da primeira autora, apresentada à Universidade Federal do Rio Grande do Sul - UFRGS. Trabalho realizado com apoio financeiro da CAPES. Trabalho apresentado no XXXI Congresso Brasileiro de Ciência do Solo, 2007 (Gramado, RS).

(2) Aluna de Pós-doutorado no Programa de Pós-Graduação em Ciência do Solo, UFRGS. Av. Bento Gonçalves, 7712, Caixa Postal 15100, CEP 90001-970, Porto Alegre (RS). E-mail: marganicolodi@hotmail.com

(3) Professores do Programa de Pós-Graduação em Ciência do Solo, UFRGS. E-mails: cgianello@hotmail.com.; ibanghi@ufrgs.br; marrej@portoweb.com.br; mieln@ufrgs.br.
} 


\title{
SUMMARY: INSUFFICIENCY OF THE MINERALIST CONCEPT TO EXPRESS SOIL FERTILITY AS PERCEIVED BY PLANTS IN NO-TILLAGE SYSTEM
}

\begin{abstract}
Soil fertility is defined as the capacity of the soil to supply nutrients, in a balanced proportion for plant growth and productivity, in the absence of toxic elements. This concept, based on the mineralist theory, is widely used around the world. With the shift away from conventional systems, high crop yields are frequently obtained under no-tillage, even where soil fertility indexes are considered inadequate, according to soil fertilizer recommendations for the conventional system. It is possible that the traditional evaluation, and consequently, the mineralist concept might be inadequate or insufficient to express soil fertility as perceived by plants grown in no-tillage systems in the long term. The objective of this study was to verify the capacity of the mineralist concept and its evaluation system, to express fertility as perceived by plants in a no-tillage system with different crop rotations. Soil fertility indicators and grain corn yields were evaluated in the growing season 2005/06, in two irrigated experiments conducted for 20 years in Eldorado do Sul, State of Rio Grande do Sul - Brazil, in different cultivation systems. The results showed that soil chemical indicators, commonly used to evaluate soil fertility, could not always detect changes in the soil productivity according to the different crop management systems. The tested soil fertility indexes were generally weakly associated with corn yield in this evaluation, which shows that the mineralist concept of soil fertility is not sufficient to express the fertility perceived by plants grown in soils of long-term no-tillage systems.
\end{abstract}

Index terms: chemical indexes, corn yield, long term no-tillage system.

\section{INTRODUÇÃO}

Fertilidade do solo, conceitualmente, é sua capacidade de fornecer nutrientes, em quantidade e proporção adequadas às plantas, na ausência de elementos tóxicos, para o seu desenvolvimento e produtividade; essencialmente, um conceito restrito às condições químicas do solo. Porém, o termo "fertilidade" significa produzir abundantemente (Wikipédia, 2006). Este conceito, baseado na teoria mineralista - os minerais (nutrientes) solúveis são o alimento das plantas — construída por Liebig (1842), não foi alterado e é amplamente utilizado no mundo há mais de um século e meio (Scarponi, 1949). A avaliação da fertilidade do solo, definida com base no conceito mineralista, é feita de modo semelhante em todo o Brasil, pela interpretação dos resultados de alguns indicadores analisados quimicamente em amostras de solo (Anghinoni, 2005). Com base nos resultados dessa avaliação, são recomendados fertilizantes, adubos (minerais ou orgânicos) e corretivos (de acidez ou de alcalinidade) para aumentar ou manter a fertilidade dos solos e, conseqüentemente, potencializar a produtividade das culturas.

Os solos, desde o início da agricultura intensiva no Brasil, têm sido cultivados no sistema convencional (SC) - preparados com arado e grade de discos, e a palha (resíduos das culturas) queimada, seguindo a tradição européia e americana. Na década de 1960, iniciou-se no Sul do Brasil, com grande expansão somente a partir da década de 1980, o cultivo no sistema plantio direto (SPD) em que o solo é resolvido somente na linha de semeadura, a superfície é mantida coberta pela palha e é feita rotação de culturas (Denardin, 1998). A diferença fundamental que se verifica num mesmo solo cultivado no SC e no SPD é, respectivamente, a destruição freqüente e a preservação das relações construídas no solo com o tempo de cultivo nesses sistemas. Isso se deve, principalmente, às diferenças no grau e intensidade de revolvimento do solo e no manejo da palha e de diversidade biológica desses sistemas que determinam as condições dadas pelo solo para o crescimento e desenvolvimento das plantas. No SC, o solo é totalmente desagregado pelo revolvimento, até duas vezes por ano; isto, associado à queima da palha, promove rápida degradação da matéria orgânica (MO) e considerável alteração da biologia do solo. Por isso no SC, em que as condições físicas e biológicas do solo são pouco alteradas no tempo, as culturas respondem bem à melhoria das condições químicas, principalmente as alteradas pela aplicação dos fertilizantes. Entretanto, as relações construídas no solo com o tempo de cultivo no SPD são preservadas e enriquecidas pela diversidade de espécies utilizadas na rotação. Por isso, no SPD, além das condições químicas, são alteradas e melhoradas, também, as físicas e biológicas do solo.

Nos solos cultivados no SC, a aplicação do conceito mineralista, por meio da avaliação e da recomendação de fertilizantes, produz resultados satisfatórios. Em geral, não são obtidas altas produtividades quando os indicadores de fertilidade 
- pH, fósforo $(\mathrm{P})$ e potássio (K) disponíveis, MO e saturação por bases (V) - são baixos ou quando a saturação por alumínio $(\mathrm{m})$ e o teor de alumínio trocável $\left(\mathrm{Al}_{\text {troc }}\right)$ são altos. No entanto, nos últimos anos, inúmeros resultados têm mostrado que altas produtividades podem ser obtidas em solos cultivados no SPD com valores de $\mathrm{pH}, \mathrm{m}, \mathrm{V}$ e teores de $\mathrm{Al}_{\text {troc }}$ (Pöttker \& Ben, 1998; Salet, 1998; Caires et al., 1999) e P disponível (Sá, 2004) considerados inadequados para um bom crescimento das plantas no SC. Por exemplo, no $\mathrm{SPD}$, a produtividade não foi limitada em solo com $\mathrm{pH}$ em água de 4,7 e 2,3 $\mathrm{cmol}_{\mathrm{c}} \mathrm{dm}^{-3}$ de $\mathrm{Al}_{\text {troc }}$ (Pöttker \& Ben, 1998), nem com $18 \%$ de m e $32 \%$ de V (Caires et al., 1999). Esses dados permitem questionar se os valores ou faixas de interpretação de indicadores químicos, utilizados na avaliação e recomendação de fertilizantes no Brasil, representam, com confiabilidade, a fertilidade de um solo e garantem a expressão do potencial produtivo de uma cultura em determinado ambiente. E possível que a avaliação e, conseqüentemente, este conceito sejam insuficientes para expressar a fertilidade nos solos cultivados há longo tempo no SPD. Isso conduz à reflexão sobre a adequabilidade da avaliação da fertilidade e do seu conceito mineralista para todos os sistemas de cultivo. O objetivo do trabalho foi avaliar a capacidade do conceito mineralista em expressar a fertilidade percebida pelas plantas em solo cultivado por longo período de tempo no SPD com diferentes rotações de culturas.

\section{MATERIAL E MÉTODOS}

As avaliações foram feitas em dois experimentos irrigados, conduzidos há mais de 20 anos em Eldorado do Sul (Figura 1), na Estação Experimental Agronômica da UFRGS, num Argissolo Vermelho distrófico (PVd), na safra de verão 2005/2006. Os experimentos, "Cobertura vegetal como alternativa de conservação do solo" (Experimento 1) e "Preparos de solo e cobertura vegetal como alternativa de conservação do solo" (Experimento 2), foram instalados em 1983 e 1985, respectivamente. $\mathrm{O}$ delineamento experimental foi o de blocos ao acaso, com os tratamentos distribuídos em parcelas subsubdivididas com três repetições.

No Experimento 1, compactação e descompactação do solo constituem as parcelas principais ( $16 \times 50 \mathrm{~m})$; as rotações de culturas, as subparcelas (5 x $16 \mathrm{~m})$; e a adubação nitrogenada (0 e $\left.180 \mathrm{~kg} \mathrm{ha}^{-1}\right)$, as subsubparcelas $(5 \times 8 \mathrm{~m})$. Por não se observar mais efeito da descompactação, a partir da safra 2005/2006, as subparcelas $(5 \times 16 \mathrm{~m})$ foram consideradas parcelas e as subsubparcelas, subparcelas $(5 \times 8 \mathrm{~m})$. Estas foram divididas (5 x $4 \mathrm{~m}$ ) e, nas subsubparcelas com zero de $\mathrm{N}$, foram aplicadas doses 0 e $60 \mathrm{~kg} \mathrm{ha}^{-1} \mathrm{e}$, naquelas com $180 \mathrm{~kg} \mathrm{ha}^{-1}$, foram aplicadas doses de 120 e $180 \mathrm{~kg} \mathrm{ha}^{-1}$.

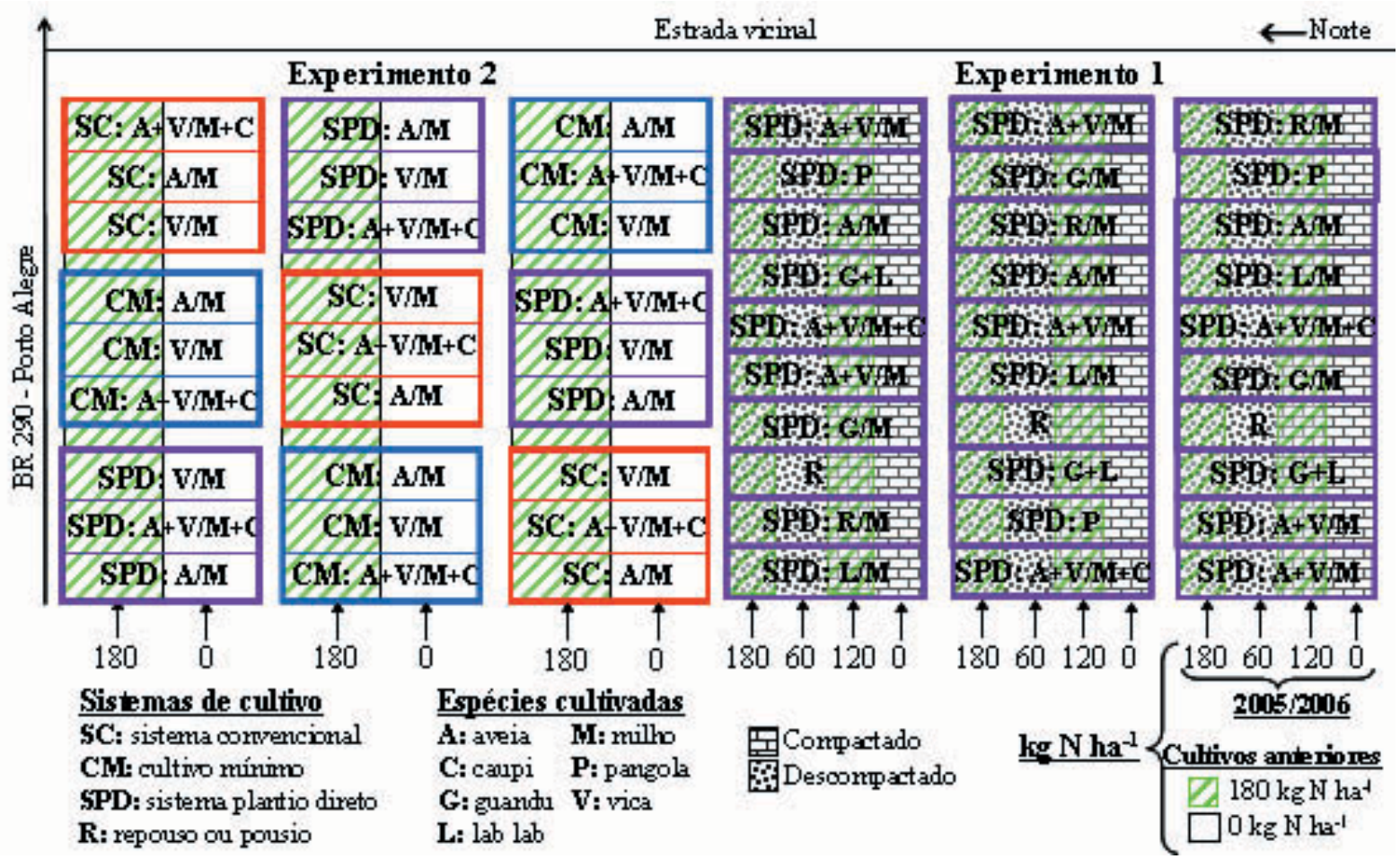

Figura 1. Representação esquemática dos experimentos conduzidos há mais de 20 anos em Eldorado do Sul (RS), na Estação Experimental Agronômica da UFRGS, em Argissolo Vermelho distrófico (PVd), avaliados neste trabalho, na safra 2005/2006: Experimento 1 "Cobertura vegetal como alternativa de conservação do solo" e Experimento 2 "Preparos de solo e cobertura vegetal como alternativa de conservação do solo". 
No Experimento 2, os sistemas de cultivo constituem as parcelas principais (15 x $20 \mathrm{~m})$; as rotações de culturas, as subparcelas (5 x $20 \mathrm{~m}$ ); e a adubação nitrogenada (0 e $\left.180 \mathrm{~kg} \mathrm{ha}^{-1}\right)$, as subsubparcelas $(5 \times 10 \mathrm{~m})$.

$\mathrm{Na}$ instalação dos experimentos, foram feitas a calagem e a adubação corretiva; os insumos foram incorporados ao solo com lavra até $20 \mathrm{~cm}$ de profundidade em toda a área. No Experimento 2, a calagem foi feita novamente em 1988, 1992 e 1996.

Em todos os tratamentos, dos dois experimentos (Figura 1), exceto nas parcelas de cultivo mínimo no Experimento 2, foram avaliados indicadores da fertilidade do solo e rendimento de grãos de milho. As amostras de solo foram coletadas na camada 0$10 \mathrm{~cm}$ na segunda quinzena de outubro de 2005, compostas por duas subamostras. As subamostras foram coletadas com espátula e consistiram em uma fatia de solo de $5 \mathrm{~cm}$ de espessura e $10 \mathrm{~cm}$ de largura. Para a determinação de $\mathrm{N}$ mineral, $20 \mathrm{~mL}$ de solo foram coletados e imediatamente transferidos para frascos de vidro que continham $100 \mathrm{~mL}$ de solução $1 \mathrm{~mol} \mathrm{~L}{ }^{-1}$ de $\mathrm{KCl}$, e mantidos em $4{ }^{\circ} \mathrm{C}$ até a determinação. O milho (Pioneer 32R21) foi semeado na primeira quinzena de novembro de 2005. A adubação, feita na linha de semeadura, foi de 250 $\mathrm{kg} \mathrm{ha}{ }^{-1}$ da fórmula 00-20-20 (N- $\left.\mathrm{P}_{2} \mathrm{O}_{5}-\mathrm{K}_{2} \mathrm{O}\right)$; em cobertura foi aplicado $1 / 3$ da quantidade da adubação nitrogenada (uréia) prevista para cada tratamento (Figura 1) no estádio fenológico V4 e 2/3 no V6. Nas amostras de solo, foram determinados o $\mathrm{pH}$ em água, os teores de $\mathrm{P}$ e $\mathrm{K}$ disponíveis (Mehlich 1), de Ca, $\mathrm{Mg}$ e Al trocáveis de $\mathrm{MO}$ e de $\mathrm{N}$ mineral e total (TKN) conforme metodologia descrita em Tedesco et al. (1995). Os valores V, m e CTC efetiva foram obtidos por cálculo. O rendimento de grãos foi avaliado em área de 5,4 e 7,2 $\mathrm{m}^{2}$ por tratamento nos experimentos 1 e 2 , respectivamente, cujo peso foi convertido em tha ${ }^{-1}$.

$\mathrm{O}$ conceito mineralista da fertilidade estabelece uma relação direta entre os teores de nutrientes no solo e o rendimento das culturas, quando os outros fatores de crescimento, dentre eles a eliminação da toxidez e o suprimento adequado de água, são adequados. Na prática, estabeleceram-se valores ou relações para determinados indicadores e, a partir da avaliação desses, definiram-se as necessidades de adequação dos mesmos para determinada cultura ou grupos de culturas. Assim, para os macronutrientes $\mathrm{P}, \mathrm{K}, \mathrm{Ca}, \mathrm{Mg}$ e alguns micronutrientes, foram estabelecidos valores conhecidos como teores/níveis críticos ou faixas adequadas ou de suficiência (Raij et al., 1997; CQFSRS/SC, 2004; Souza \& Lobato, 2004). Para a acidez do solo, foram estabelecidos valores de $\mathrm{pH}, \mathrm{V}, \mathrm{m}$ e de teor de $\mathrm{Al}_{\text {troc }}$ como critérios de recomendação ou não de calagem para os solos. Portanto, são clássicas as relações entre $\mathrm{pH}$ e $\mathrm{Al}_{\text {troc }}$, $\mathrm{pH}$ e V e aumento de $\mathrm{pH}$ e incremento de CTC efetiva. É consenso que solos cujos valores dos indicadores de fertilidade estão fora das faixas adequadas, em geral, produzem menos ou não produzem, enquanto solos considerados de fertilidade adequada, e tendo os outros fatores de crescimento também adequados, tendem a produzir próximo do seu potencial máximo. Neste trabalho, a capacidade do conceito mineralista, descrito anteriormente, em expressar a fertilidade do solo percebida pelas plantas em sistemas diferentes do SC, é questionada pela comparação entre as relações clássicas de indicadores e entre estes e o rendimento de grãos de milho. As relações apresentadas são entre $\mathrm{pH}$ e $\mathrm{Al}_{\text {troc }}, \mathrm{V}$ e CTC efetiva; $\mathrm{Al}_{\text {troc }}$ e o valor $\mathrm{m}$ e teor de $\mathrm{MO}$; e $\mathrm{pH}, \mathrm{Al}_{\text {troc }}, \mathrm{V}$, teor de $\mathrm{P}$, de $\mathrm{K}$ e de $\mathrm{MO}$ e rendimento de grãos.

\section{RESULTADOS E DISCUSSÃO}

Os resultados dos experimentos conduzidos há mais de 20 anos demonstram que as tendências centrais de algumas das relações clássicas entre os indicadores de fertilidade não se alteraram com a mudança do SC para o SPD (Figura 2). Isso pode ser verificado para a relação $\mathrm{Al}_{\text {troc }}$ e $\mathrm{pH}$ (Figura 2a). À medida que este aumentou, aquele diminuiu, corroborando os resultados de muitos trabalhos (Coelho, 1973; Kaminski, 1974 entre outros). Entretanto, os resultados obtidos nos tratamentos em SC distribuem-se mais à direita da figura $2 \mathrm{a}$, enquanto os obtidos no SPD, além de apresentarem maior dispersão, formam uma faixa ampla e distribuem-se mais à esquerda. Essa diferença na distribuição indica que, em um mesmo solo, para valores iguais de $\mathrm{pH}$, o teor de $\mathrm{Al}_{\text {troc }}$ pode ser diferente, dependendo do sistema de cultivo e da rotação de culturas adotados. Em geral, no SPD, o teor de $\mathrm{Al}_{\text {troc }}$ é menor e seu efeito menos pronunciado sobre a planta do que no SC, para um mesmo valor de $\mathrm{pH}$. Isso, associado aos benefícios do SPD, permite o crescimento das culturas a um $\mathrm{pH}$ mais baixo neste sistema do que naquele.

No SC, produtividades elevadas para a maioria das culturas somente são obtidas com valores de pH em água entre 5,5 e 6,5 e na ausência de $\mathrm{Al}_{\text {troc }}$ no solo (Coelho, 1973; Quaggio, 2000; CQFSRS/SC, 2004). Entretanto, neste trabalho, isso não foi verificado. $\mathrm{O}$ rendimento médio de grãos de milho não diminuiu com o aumento de $\mathrm{Al}_{\text {troc }}$ e a diminuição do $\mathrm{pH}$. Nos tratamentos em que o $\mathrm{pH}$ era $\leq 4,6$ e o $\mathrm{Al}_{\text {troc }} \geq 1,2 \mathrm{cmol}_{\mathrm{c}} \mathrm{dm}^{-3}$ (Figura 2a, conjunto de pontos circulado em laranja), o rendimento médio foi de 7,2 t ha' ${ }^{-1}$ em pH $\leq 5,1$ e $\mathrm{Al}_{\text {troc }} \geq 0,6 \mathrm{cmol}_{\mathrm{c}} \mathrm{dm}^{-3}$ (Figura 2a, conjunto de pontos circulado em preto), foi de $6,4 \mathrm{tha}^{-1} ; \mathrm{em} \mathrm{pH} \leq 5,3 \mathrm{e} \mathrm{Al}_{\text {troc }} \geq 0,3 \mathrm{cmol}_{\mathrm{c}} \mathrm{dm}^{-3}$ (Figura 2a, conjunto de pontos circulado em rosa), foi de $7,1 \mathrm{tha}^{-1}$. O menor rendimento médio $(4,7 \mathrm{t}$ ha $^{-1}$ ) foi obtido no tratamento com $\mathrm{pH} \geq 5,5$ e zero de $\mathrm{Al}_{\text {troc }}$ (Figura 2a, conjunto de pontos circulado em azul). 

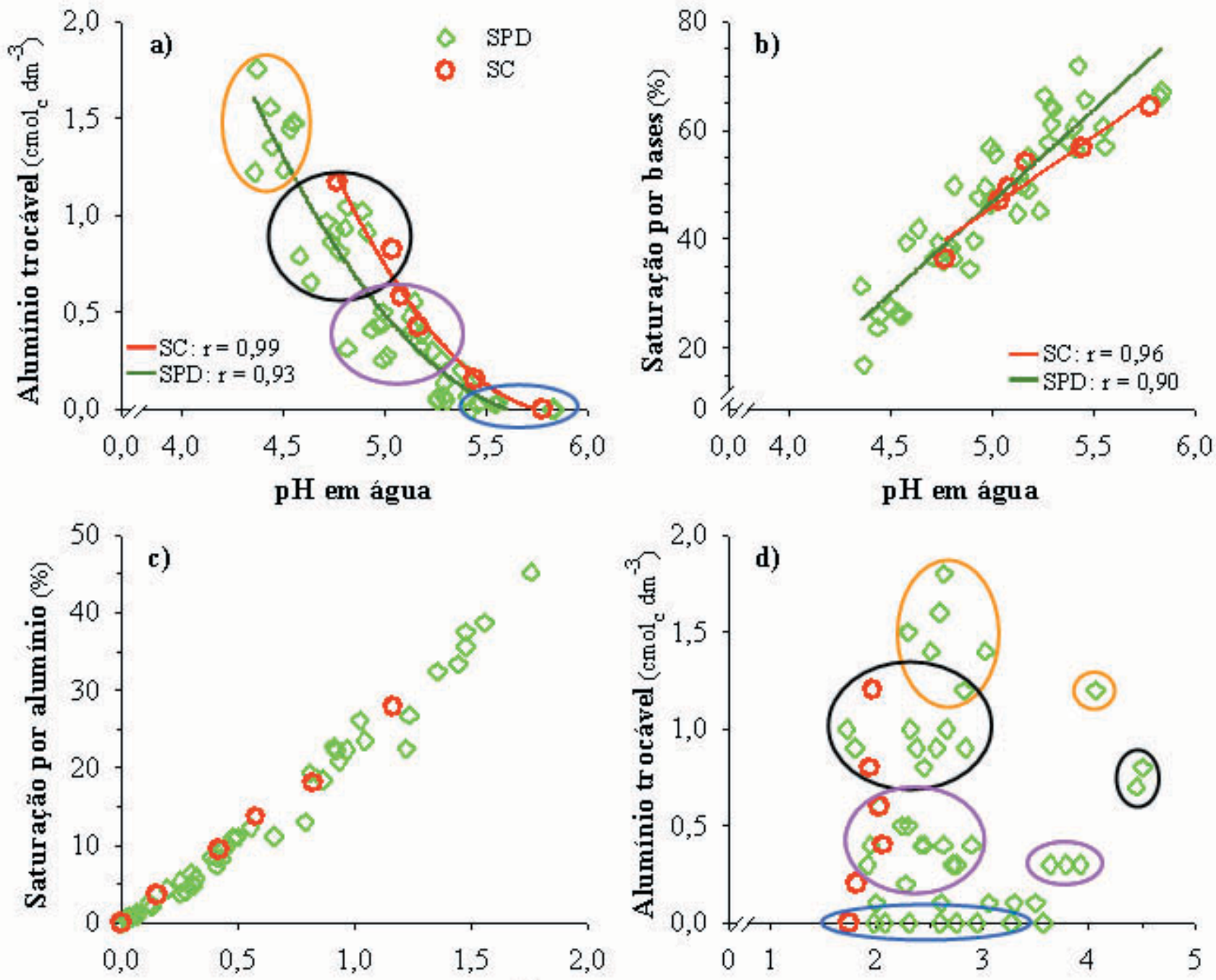

Alumínio trocável $\left(\mathrm{cmol}_{\mathrm{c}} \mathrm{dm}^{-3}\right)$
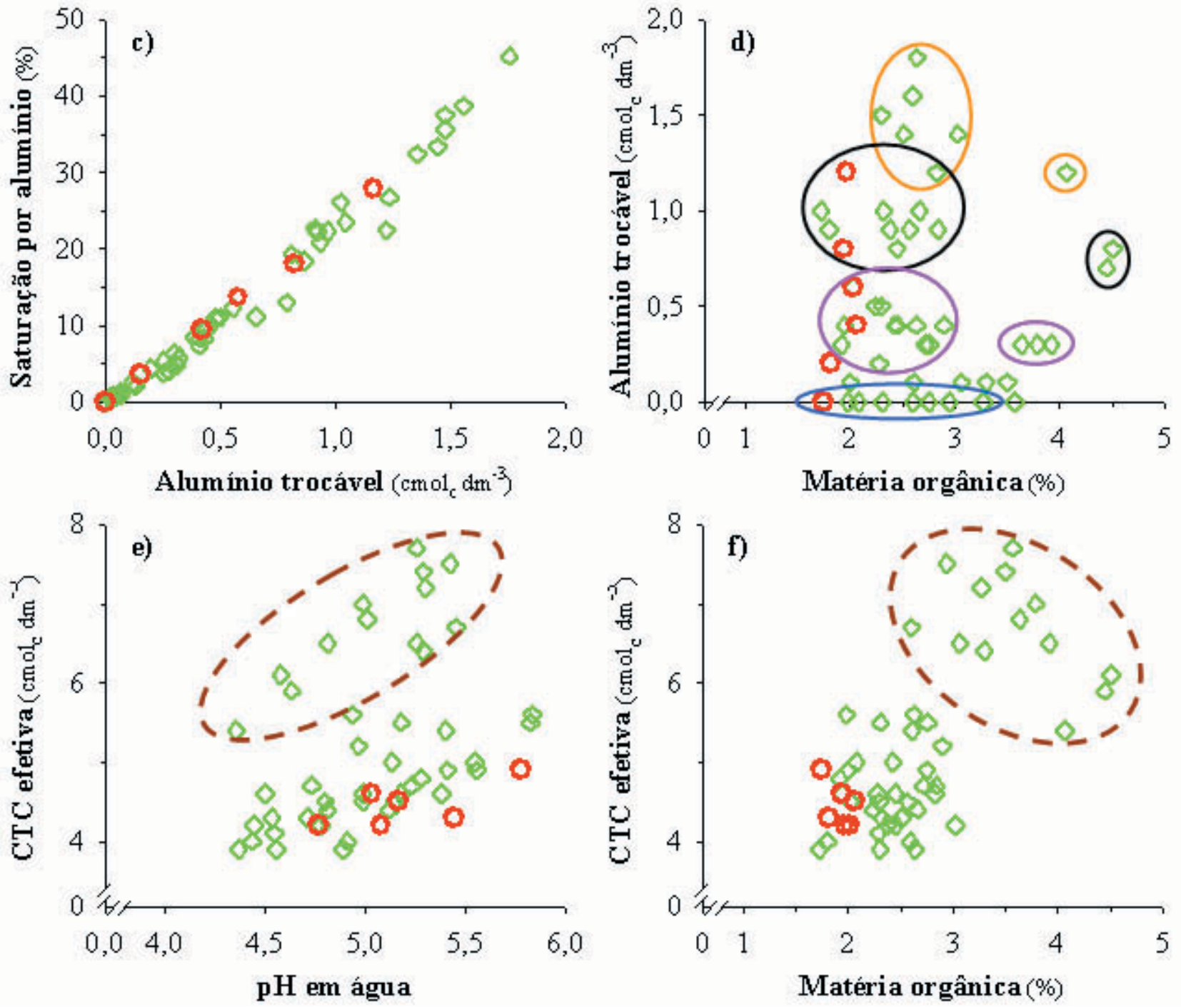

Figura 2. Relações clássicas entre indicadores tradicionais da fertilidade do solo avaliados em experimentos com diferentes históricos de cultivo, conduzidos há mais de 20 anos em Eldorado do Sul (RS) (PVd, amostrado na camada de $0-10 \mathrm{~cm})$. 
A relação entre $\mathrm{pH}$ e $\mathrm{V}$ (Figura $2 \mathrm{~b}$ ) em solo cultivado no SC e no SPD é semelhante. No entanto, observa-se que o valor $\mathrm{V}$ é maior no SPD do que no $\mathrm{SC}$ para um mesmo $\mathrm{pH}$. A um valor $\mathrm{V}$ de $65 \%$, correspondem valores de $\mathrm{pH}$ de 5,5 no SPD e 5,8 no SC. Esses dados são concordantes com os utilizados pela CQFS-RS/SC (2004) para estabelecer o critério 5,5 de $\mathrm{pH}$ e $65 \%$ de $\mathrm{V}$ para recomendar calagem no SPD. Essas diferenças, devidas aos sistemas de cultivo, não foram observadas entre $\mathrm{Al}_{\text {troc }}$ e $\mathrm{m}$ (Figura 2c).

A MO, principalmente por seus ligantes orgânicos, tem a capacidade de complexar o $\mathrm{Al}_{\text {troc }}$, reduzindo sua atividade na solução do solo e, conseqüentemente, diminuindo sua toxidez para as plantas (Salet, 1998). Isso indica que no SPD poderia haver uma relação inversa entre $\mathrm{MO}$ e $\mathrm{Al}_{\text {troc }}$. Entretanto, nenhuma tendência clara foi observada nesses experimentos (Figura 2d). Provavelmente, não há uma relação entre teor de $\mathrm{MO}$ total do solo e $\mathrm{Al}_{\text {troc }}$, mas, sim, entre este e os componentes lábeis da MO, cuja meiavida é curta no solo, porém se formam constantemente no SPD, em quantidade e qualidade variáveis com as rotações de culturas adotadas. Assim, solos no SPD com valores altos de $\mathrm{Al}_{\text {troc }}$, mas na presença de altos teores de $\mathrm{MO}$, podem não restringir o crescimento de muitas culturas, embora restrinjam no SC. Em algumas rotações no SPD, o teor de MO duplicou em 20 anos (Figura 2d). Podese observar, no entanto, que, nos tratamentos com teor de $\mathrm{Al}_{\text {troc }}$ maior (Figura 2a, conjunto de pontos circulado em laranja), o rendimento médio de grãos foi o mais alto $\left(7,2 \mathrm{t} \mathrm{ha}^{-1}\right)$ para um teor de $\mathrm{MO}$, à exceção de um tratamento, menor que 3 \% (Figura $2 \mathrm{~d}$, conjunto de pontos circulado em laranja). Portanto, os maiores rendimentos não foram proporcionais ao maior teor de MO.

Dentre as relações clássicas de indicadores da fertilidade, destacam-se, ainda, aquelas entre $\mathrm{pH}$ e CTC efetiva e entre esta e MO (Figuras 2e e 2f). Em geral, a CTC efetiva aumenta à medida que se corrige a acidez do solo (o pH aumenta) (Volkweiss, 1989). Isso pode ser observado para o conjunto de pontos que representa o SC (Figura 2e). Embora de modo pouco acentuado, a CTC efetiva aumentou de 4,2 $\mathrm{cmol}_{\mathrm{c}} \mathrm{dm}^{-3}$ para $4,7 \mathrm{cmol}_{\mathrm{c}} \mathrm{dm}^{-3}$, com o aumento do $\mathrm{pH}$ de 4,8 para 5,8 . Contudo, na análise conjunta de todos os tratamentos avaliados, verifica-se que há grande dispersão de pontos, o que não permite definir claramente a relação entre pH e CTC efetiva. Nesse caso, o efeito da rotação de culturas é maior que o efeito do aumento de $\mathrm{pH}$. Se os pontos da figura $2 \mathrm{e}$ forem agrupados em três conjuntos (SC, SPD e SPD circulado), é possível afirmar que para cada conjunto em separado, há correspondência entre $\mathrm{pH}$ e CTC efetiva. No entanto, valores médios de 5,2, 5,0 e 5,0 de $\mathrm{pH}$, para o conjunto SC, SPD e SPD circulado, correspondem à CTC efetiva de 4,5, 4,6 e 6,7 $\mathrm{cmol}_{\mathrm{c}}$ $\mathrm{dm}^{-3}$, respectivamente, ou seja, não há variação da CTC efetiva em razão da variação do $\mathrm{pH}$ do solo. Na figura 2e, o conjunto de pontos destacado com um círculo corresponde ao destacado na figura $2 \mathrm{f}$. $\mathrm{Na}$ figura $2 \mathrm{f}$, observa-se que a $\mathrm{MO}$ do solo aumentou à medida que a CTC efetiva diminuiu. Pela relação entre MO e CTC efetiva (Figura 2f), em termos médios, pode-se observar para o conjunto do SC que $1,9 \%$ de $\mathrm{MO}$ corresponde a $4,5 \mathrm{cmol}_{\mathrm{c}} \mathrm{dm}^{-3} \mathrm{de}$ CTC efetiva; para o SPD não circulado, $2,3 \%$ a $4,6 \mathrm{cmol}_{\mathrm{c}} \mathrm{dm}^{-3}$; para o SPD circulado, $3,6 \%$ a 6,7 $\mathrm{cmol}_{\mathrm{c}} \mathrm{dm}^{-3}$. Isso indica que a ação benéfica da MO, em alguns casos, está mais relacionada com o tipo e qualidade do que com sua quantidade.

$\mathrm{Na}$ figura 3 , são comparados indicadores da fertilidade do solo com o rendimento de grãos, utilizando todos os dados obtidos nos dois experimentos. Nas comparações feitas para um determinado tratamento em uma figura, há sempre a correspondência de pontos nos demais. Alguns dos resultados apresentados nas figuras, para clareza de entendimento, são reapresentados no Quadro 1.

A inadequabilidade de alguns indicadores da fertilidade do solo utilizados no SC para o SPD pode ser observada pelas relações entre os indicadores tradicionais e o rendimento das culturas (Figura 3 ). O rendimento, quando os demais fatores que influenciam o crescimento estão em condições adequadas (irrigação, controle de pragas, etc.), é proporcional ao teor de nutrientes - sendo determinante o que estiver em condição mínima e, ou, à eliminação da acidez ou alcalinidade excessiva. Assim, pelo conceito mineralista da fertilidade, esperar-se-ia, nas condições descritas, um rendimento de no mínimo $90 \%$ do máximo, quando os valores de $\mathrm{pH}$ e o teor dos nutrientes fossem interpretados como adequados (CQFS-RS/ $\mathrm{SC}, 2004)$. Nesses experimentos, isso corresponde, para o SC, na rotação A/M (Quadro 1; pontos destacados com quadrado rosa na Figura 3). Embora baixo, se comparado com os outros, o teor de $\mathrm{P}$ deste tratamento é adequado (faixa adequada para esta classe textural é $12-18 \mathrm{mg} \mathrm{dm}^{-3}$, segundo a CQFS-RS/SC, 2004). Na comparação entre esse tratamento e o SPD-A/M - semelhante nos valores dos indicadores, porém, em SPD há mais de 20 anos -, observa-se um rendimento de aproximadamente $50 \%$ menor na rotação $\mathrm{A} / \mathrm{M}\left(5,7 \mathrm{t} \mathrm{ha}^{-1}\right)$ em relação ao SPD-A/M (9,4 t ha $\left.{ }^{-1}\right)$ (Quadro 1; pontos destacados com quadrado azul na Figura 3). Obviamente, nesse caso, o fator de maior influência foi o sistema de cultivo, que alterou o potencial produtivo do solo. Portanto, a interpretação dos resultados analíticos dos indicadores mantém a coerência, pois, em ambos os casos, os solos são considerados de fertilidade adequada (CQFS-RS/SC, 2004). 

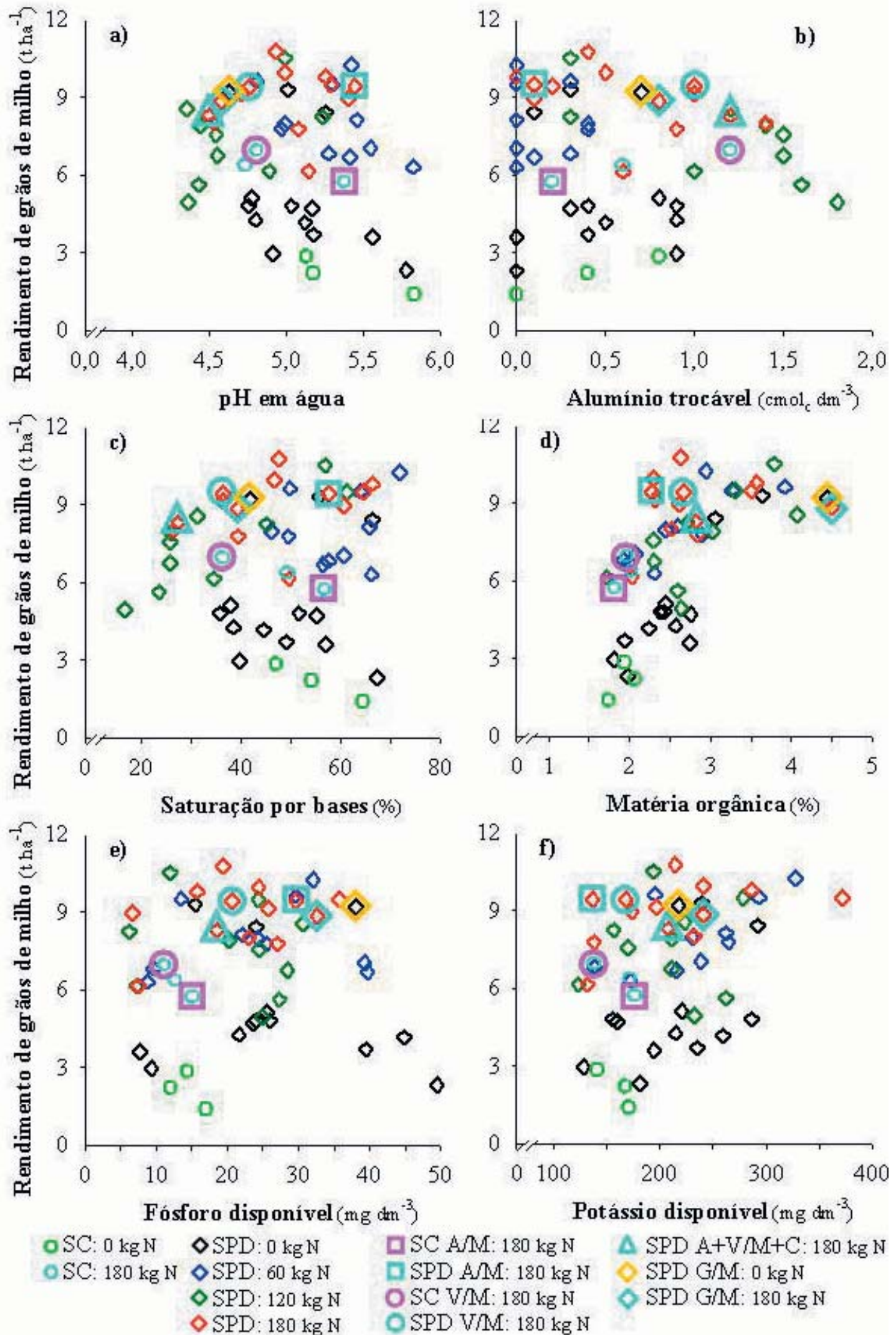

Figura 3. Relações entre o rendimento de grãos de milho e os indicadores tradicionais da fertilidade do solo avaliados em experimentos com diferentes históricos de cultivo, conduzidos há mais de 20 anos em Eldorado do Sul (RS) (PVd, amostrado na camada de 0-10 cm). 
Quadro 1. Rendimento de grãos de milho e resultados dos principais indicadores da fertilidade do solo ${ }^{(1)}$ de alguns tratamentos avaliados, na safra de 2005/2006, nos experimentos conduzidos há mais de 20 anos em Eldorado do Sul (RS)

\begin{tabular}{|c|c|c|c|c|c|c|c|c|c|c|}
\hline $\begin{array}{l}\text { Sistema } \\
\text { de cultivo }\end{array}$ & $\begin{array}{c}\text { Rotação de } \\
\text { culturas }\end{array}$ & $\begin{array}{c}\text { Adubação } \\
\text { nitrogenada }\end{array}$ & $\begin{array}{l}\text { Rendimento } \\
\text { de grãos }\end{array}$ & $\begin{array}{c}\text { pH } \\
\text { água }\end{array}$ & $\mathrm{Al}_{\text {troc }}$ & $\mathbf{m}$ & $\frac{P}{\text { disp }}$ & $\frac{K}{\text { yeis }}$ & MO & V \\
\hline & & $\mathrm{kg} \mathrm{ha}^{-1}$ de N & $\mathrm{tha}^{-1}$ & & $\mathrm{cmol}_{\mathrm{c}} \mathrm{dm}^{-3}$ & $\%$ & \multicolumn{2}{|c|}{$-\mathrm{mg} \mathrm{dm}^{-3}-$} & — $\%$ & 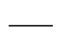 \\
\hline $\mathrm{SC}$ & $\mathrm{A} / \mathrm{M}$ & 180 & 5,7 & 5,4 & 0,2 & 4 & 15 & 176 & 1,8 & 57 \\
\hline SPD & $\mathrm{A} / \mathrm{M}$ & 180 & 9,4 & 5,4 & 0,2 & 4 & 30 & 136 & 2,3 & 58 \\
\hline $\mathrm{SC}$ & $\mathrm{V} / \mathrm{M}$ & 180 & 6,9 & 4,8 & 1,2 & 28 & 11 & 139 & 2,0 & 36 \\
\hline SPD & $\mathrm{V} / \mathrm{M}$ & 180 & 9,4 & 4,8 & 1,0 & 24 & 21 & 168 & 2,7 & 36 \\
\hline SPD & $\mathrm{A}+\mathrm{V} / \mathrm{M}+\mathrm{C}$ & 180 & 8,3 & 4,5 & 1,2 & 27 & 19 & 207 & 2,8 & 27 \\
\hline SPD & G/M & 0 & 9,2 & 4,6 & 0,7 & 11 & 38 & 217 & 4,5 & 42 \\
\hline SPD & G/M & 180 & 8,8 & 4,6 & 0,8 & 13 & 33 & 241 & 4,4 & 39 \\
\hline
\end{tabular}

(1) PVd (0-10 cm); classe textural 2 (20 a $40 \%$ de argila) (CQFS-RS/SC, 2004).

A comparação entre sistemas e entre rotações dentro de sistemas pode ser feita com os tratamentos SC-A/M, SC-V/M e SPD-A/M e SPDV/M (Quadro 1). No SC, houve incremento de rendimento de aproximadamente $20 \%$ entre o SC$\mathrm{A} / \mathrm{M}\left(5,7 \mathrm{t} \mathrm{ha}^{-1}\right)$ e o $\mathrm{SC}-\mathrm{V} / \mathrm{M}\left(6,9 \mathrm{t} \mathrm{ha}^{-1}\right)$. O resultado poderia ser considerado normal, caso os valores dos indicadores da fertilidade fossem semelhantes. Assim, o efeito seria atribuído à rotação. No entanto, o pH diminuiu de 5,4 para 4,8, o valor $\mathrm{V}$ diminuiu de $57 \%$ para $36 \%$ e o valor de $\mathrm{m}$ passou de $4 \%$ para $28 \%$ no tratamento SC-V/M. Pelo conceito mineralista - fornecimento de nutrientes e ausência de elementos tóxicos -, nesse tratamento, a fertilidade era adequada e tornou-se baixa. Logo, para expressar seu potencial, esse solo deveria ter a fertilidade corrigida. Nesse caso, para o próprio SC, quando forem utilizadas diferentes rotações de culturas por períodos longos, o sistema atual de avaliação da fertilidade do solo pode não ser adequado.

Os resultados obtidos nos tratamentos SPD-A+V/ $\mathrm{M}+\mathrm{C}$ (Quadro 1; pontos destacados com triângulo azul na Figura 3) e SPD-G/M com e sem adubação nitrogenada (Quadro 1; pontos destacados com quadrilátero azul e laranja, respectivamente, na Figura 3) indicam ser possível a obtenção de altos rendimentos, quando a fertilidade do solo é interpretada, tradicionalmente, como baixa (Raij et al., 1997; CQFS-RS/SC, 2004; Souza \& Lobato, 2004). No tratamento SPD-A+V/M+C, merecem a atenção o rendimento de 8,3 $\mathrm{t} \mathrm{ha}^{-1}$ de grãos, o $\mathrm{pH}$ do solo baixo, o $\mathrm{m}$ e o teor de $\mathrm{Al}_{\text {troc }}$ altos e a $\mathrm{V}$ baixa. Nos tratamentos SPD-G/M, com ou sem N, não houve efeito deste no rendimento. Embora a fertilidade nesses tratamentos também seja interpretada como baixa, os rendimentos obtidos foram muito altos (Quadro 1).
Os resultados e as considerações apresentados mostraram que as diferenças no rendimento foram devidas mais ao histórico do cultivo, ou seja, aos sistemas de cultivo, rotação de culturas, adubação nitrogenada e interação destes do que aos valores dos indicadores químicos da fertilidade, tradicionalmente avaliados. Nem a relação entre o $\mathrm{N}$ total (Figura 4a) ou mineral (Figura 4b) no solo e o rendimento melhorou a interpretação da fertilidade percebida pela cultura do milho nos experimentos avaliados.

Nesses experimentos conduzidos há mais de 20 anos, é possível observar o grau de dificuldade que existe na avaliação da fertilidade do solo, decorrente das inúmeras combinações possíveis de sistemas de cultivo e de rotações de culturas, além do efeito do tempo de cultivo nos indicadores (Figura 3). Num sistema simples (menos complexo), poucos fatores são responsáveis pelo rendimento e a alteração de qualquer um deles gera mudanças significativas nos resultados. Em sistemas mais complexos - com o tempo, no SPD, além das condições químicas, mudam também as físicas e as biológicas; no SC, somente as químicas mudam significativamente no tempo-, em que há um número grande de fatores influenciando o rendimento e a alteração de um ou alguns deles têm pouca ou nenhuma influência (D'Agostini, 2006).

Os resultados dos experimentos mostraram que nem sempre indicadores químicos detectam a mudança na capacidade produtiva do solo promovida pelos diferentes sistemas de cultivo e rotações de culturas. Esses indicadores apresentaram baixo grau de associação com o rendimento das plantas (Figura 3). Isso se deve, em geral, à tentativa de sempre associar incremento de rendimento com incremento no teor de nutriente ou diminuição do teor de elementos tóxicos. É a visão mineralista da fertilidade do solo aplicada, em geral, com sucesso no $\mathrm{SC}$ de cultivo. 


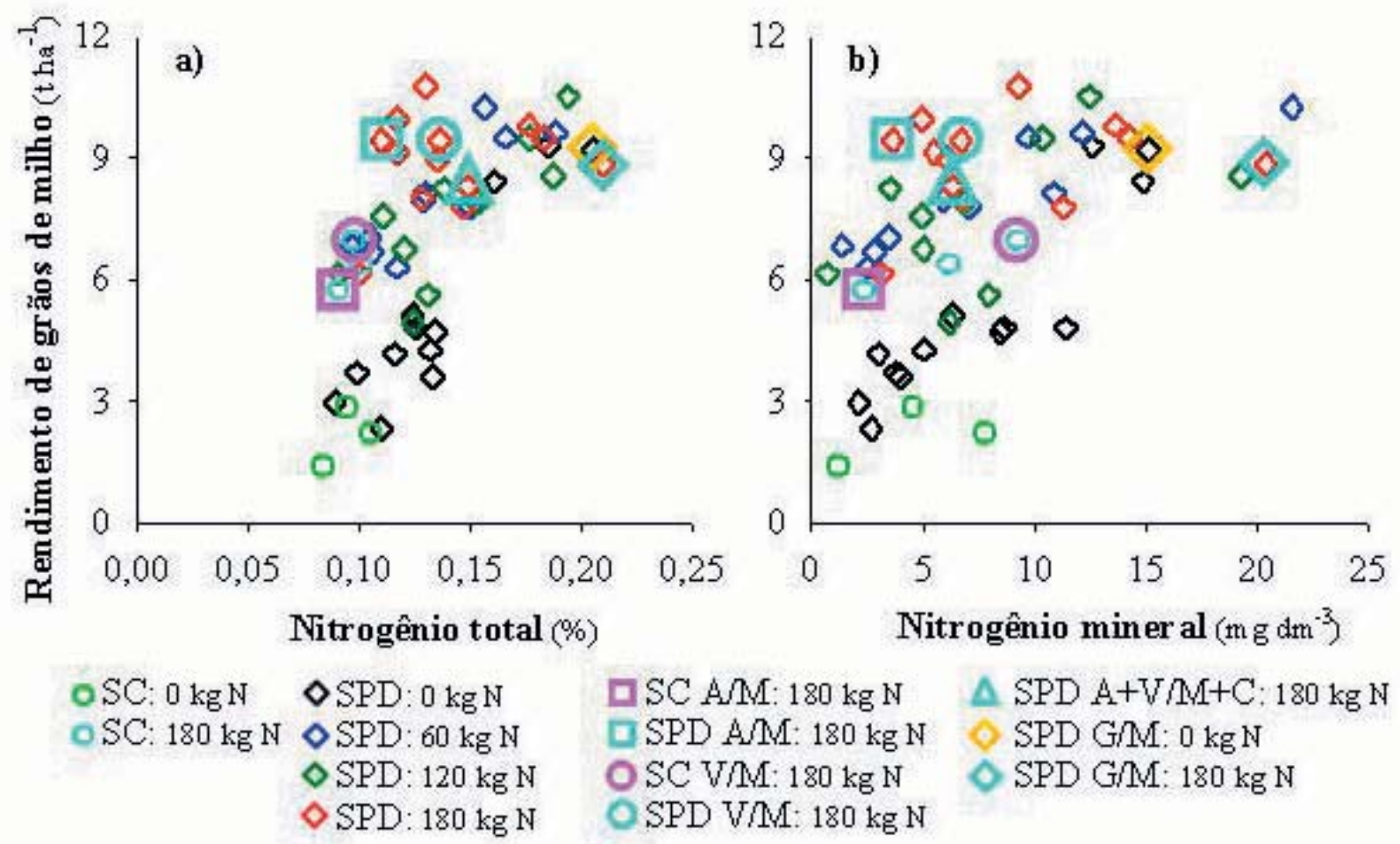

Figura 4. Relações entre o rendimento de grãos de milho e nitrogênio total (a) e mineral (b) no solo avaliados em experimentos com diferentes históricos de cultivo, conduzidos há mais de 20 anos em Eldorado do Sul (RS) (PVd, amostrado na camada de 0-10 cm).

Pelo conceito mineralista da fertilidade, não são obtidos, em geral, altos rendimentos com baixa disponibilidade de nutrientes e, ou, presença de elementos tóxicos no solo. Entretanto, esses foram obtidos em diversos tratamentos destes experimentos conduzidos há mais de 20 anos. Com a introdução de outros sistemas, dentre eles o mais difundido, o SPD, é necessário um novo conceito para a fertilidade do solo e, a partir deste, um novo sistema de avaliação. Neste, devem-se utilizar indicadores que expressem o funcionamento do solo como um todo e não apenas suas condições químicas (Nicolodi et al., 2004), a fim de estimar a capacidade produtiva desses solos, visto que o termo "fertilidade" significa produzir abundantemente (Wikipédia, 2006).

\section{CONCLUSÕES}

1. A avaliação e, conseqüentemente, o conceito mineralista da fertilidade, utilizado para definir a capacidade de um solo produzir abundantemente, são insuficientes para explicar os resultados obtidos com milho em solo cultivado no SPD por longo período com diferentes rotações de culturas.

2. A importância dos indicadores tradicionais da fertilidade do solo é menor em solos cultivados no SPD do que no SC.
3. As altas produtividades podem ser obtidas em solos cultivados por longo tempo no SPD na presença de valores de $\mathrm{Al}_{\text {troc }}$ altos e de $\mathrm{V}$ e $\mathrm{pH}$ muito baixos. São mais importantes que estes, na definição do rendimento das culturas, o tempo de cultivo do solo no SPD e a rotação de culturas adotada.

\section{LITERATURA CITADA}

ANGHINONI, I. Fertilidade do solo no ambiente subtropical. In: CONGRESSO BRASILEIRO DE CIÊNCIA DO SOLO, 30., Recife, 2005. Palestras. Recife, Sociedade Brasileira de Ciência do Slo, 2005. CD-ROM.

CAIRES, E.F.; FONSECA, A.F.; MENDES, J.; CHUEIRI, W.A. \& MADRUGA, E.F. Produção de milho, trigo e soja em função das alterações das características químicas do solo pela aplicação de calcário e gesso na superfície, em sistema de plantio direto. R. Bras. Ci. Solo, 23:315-327, 1999.

COELHO, F.S. Fertilidade do solo. 2.ed. Campinas, Instituto Campineiro de Ensino Agrícola, 1973. 384p.

COMISSÃO DE QUÍMICA E FERTILIDADE DO SOLO RS/ SC - CQFS-RS/SC. Manual de adubação e de calagem para o Estado do Rio Grande do Sul e Santa Catarina. Porto Alegre, SBCS/Núcleo Regional Sul, UFRGS, 2004. 400p. 
D'AGOSTINI, L.R. Noção de sistema: (Re)emergindo fértil em solos, fertilidade do solo (re)emergindo sistêmica. In: REUNIÃO SUL-BRASILEIRA DE CIÊNCIA DO SOLO, 6., 2006. Passo Fundo. Palestras. Passo Fundo, SBCS/ Núcleo Regional Sul, 2006. CD-ROM.

DENARDIN, J.E. Enfoque sistêmico em sistema plantio direto - Fundamentos e implicações do plantio direto nos sistemas de produção agropecuária. In: NUERNBERG, N.J. Conceitos e fundamentos do sistema plantio direto. Lages, SBCS/Núcleo Regional Sul, 1998. p.7-14.

KAMISNKI, J. Fatores de acidez e necessidade de calcário em solos do Rio Grande do Sul. Curitiba, Universidade Federal do Rio Grande do Sul, 1974. 96p. (Tese de Mestrado)

NICOLODI, M.; GIANELLO, C. \& ANGHINONI, I. Fertilidade: uma propriedade emergente do sistema solo. In REUNIÃO SUL-BRASILEIRA DE CIÊNCIA DO SOLO, 5., Florianópolis. Resumos. Florianópolis, SBCS/ Núcleo Regional Sul, 2004. CD-ROM.

PÖTTKER, D. \& BEN, J.R. Calagem em solos sob plantio direto e em campos nativos do Rio Grande do Sul. In NUERNBERG, N.J. Conceitos e fundamentos do sistema plantio direto. Lages, SBCS/Núcleo Regional Sul, 1998. p.77-92.

QUAGGIO, J.A. Acidez e calagem em solos tropicais. Campinas, Instituto Agronômico de Campinas, 2000. $111 \mathrm{p}$.
RAIJ, B.van; CANTARELLA, H.; QUAGGIO, J.A. \& FURLANI, A.M.C. Recomendações de adubação e de calagem para o Estado de São Paulo. 2.ed. Campinas, Instituto Agronômico/ Fundação IAC, 1997. 285p. (Boletim Técnico, 100)

SÁ, J.C.M. Adubação fosfatada no sistema plantio direto. In: YAMADA, T. \& ABDALLA, S.R.S. Fósforo na agricultura brasileira. Piracicaba, POTAFÓS, 2004. p.201-222.

SALET, R.L. Toxidez de alumínio no sistema plantio direto. Porto Alegre, Universidade Federal do Rio Grande do Sul, 1998. 117p. (Tese de Doutorado)

SCARPONI, F. Il concetto della fertilità nella sua evoluzione attraverso i tempi. Milano, 1949. 16p.

SOUZA, D.M.G. \& LOBATO, E. Cerrado: Correção do solo e adubação. 2.ed. Brasília, Embrapa Informação Tecnológica, 2004. 416p.

TEDESCO, M.J.; GIANELLO, C.; BISSANI, C.A.; BOHNEN, H. \& VOLKWEISS, S.J. Análise de solo, plantas e outros materiais. 2ed. Porto Alegre, Universidade Federal do Rio Grande do Sul, 1995. 174p. (Boletim Técnico, 5)

VOLKWEISS, S.J. Química da acidez dos solos. In: KAMINSKI, J.; VOLKWEISS, J. \& BECKER, F.C. SEMINÁRIO SOBRE CORRETIVOS DA ACIDEZ DO SOLO, 2., Santa Maria, 1989. Anais. Santa Maria, Universidade Federal de Santa Maria, 1989. p.7-38.

WIKIPEDIA. [Informações]. Disponível em: http:// www.wikipedia.org. Acesso em 20/11/2006. 